\title{
8 Umgang mit potenziellen Tätern bei Vorstellung in der Kinder- und Jugendpsychiatrie
}

Wie kann ein Erstgespräch mit einem potenziellen Täter geführt werden und was sollte es beinhalten? Entscheidend ist ein Zugang zu dem vorgestellten Kind oder Jugendlichen $\mathrm{zu}$ finden, insbesondere wenn eine Einweisung über PsychKG oder über den $\$ 1631$ b BGB erfolgt. Ein positiver Zugang beeinflusst maßgeblich den weiteren Behandlungsverlauf. Die Frage nach der Erreichbarkeit der potenziellen Täter beinhaltet auch, den Zugang zu ihrem „,innerworking model " und zu ihren destruktiven und oft „festgemeißelten Glaubensüberzeugungen“ zu finden. Dies gelingt nur, wenn kein „moralisierendes“Verhalten an den Tag gelegt wird und auch ihr Handeln damit nicht entschuldigt wird, dass sie eine schwere Kindheit gehabt haben. Wichtig ist, eine stressfreie Gesprächsatmosphäre mit dem potenziellen Täter herzustellen, indem man mit ihm auf seine aktuelle persönliche Krisensituation zu sprechen kommt. Aufgrund der bisherigen Erfahrungen mit potenziellen Tätern ist nach stationärer Aufnahme die Klärung folgender Fragen erforderlich:

- Wie kann ein authentischer Zugang zu dem potenziellen Täter hergestellt werden?

- Wie stark ist sein Erleben auf seine geplanten Gewalthandlungen eingeengt und wie obsessionell wird er von ihnen beherrscht?

- Ist er noch in seinen wichtigsten sozialen Kontexten mit Familie, Schule und Peer-Gruppe verankert? 
- Besteht die Gefahr, dass er von seinen bisherigen Bezugspersonen nach Bekanntgabe seiner Planungen noch stärker ausgegrenzt und abgeschrieben wird?

- Wie stark ist neben seinem Hass und seiner Rache seine Selbstmordgefährdung ausgeprägt?

Im klinischen Alltag findet der Verfasser oft einen guten Zugang zu den Jugendlichen über verschiedene Bildtechniken wie den „Belastungsrucksack“ (s. Abb. 5), um die langjährigen, aber auch aktuellen Belastungsfaktoren zu ermitteln. Kinder ab acht Jahren und Jugendliche können ihre Belastungen differenziert skalieren. Dies kann dann wie ein „Türöffner“ für das weitere Gespräch genutzt werden.

Die Belastungsfaktoren können mit dem SUD-Wert (Subjectiv Unit of Discomfort: o-10) skaliert werden. Außerdem lässt sich mit dem IUD-Wert (Intimidation Unit of Discomfort) die „Macht“ und der „Sog“ der Gewaltfantasien und des Tötungswunsches erfassen. Mit einem IUD über 5 ist der potenzielle Täter dem Einfluss seiner Vorstellungen verstärkt ausgesetzt und nicht mehr „Kapitän seiner Seele“ (Bateson 1982). Die Skalierungen können im weiteren Behandlungsverlauf im Sinne einer „Externalisierung“ genutzt werden.
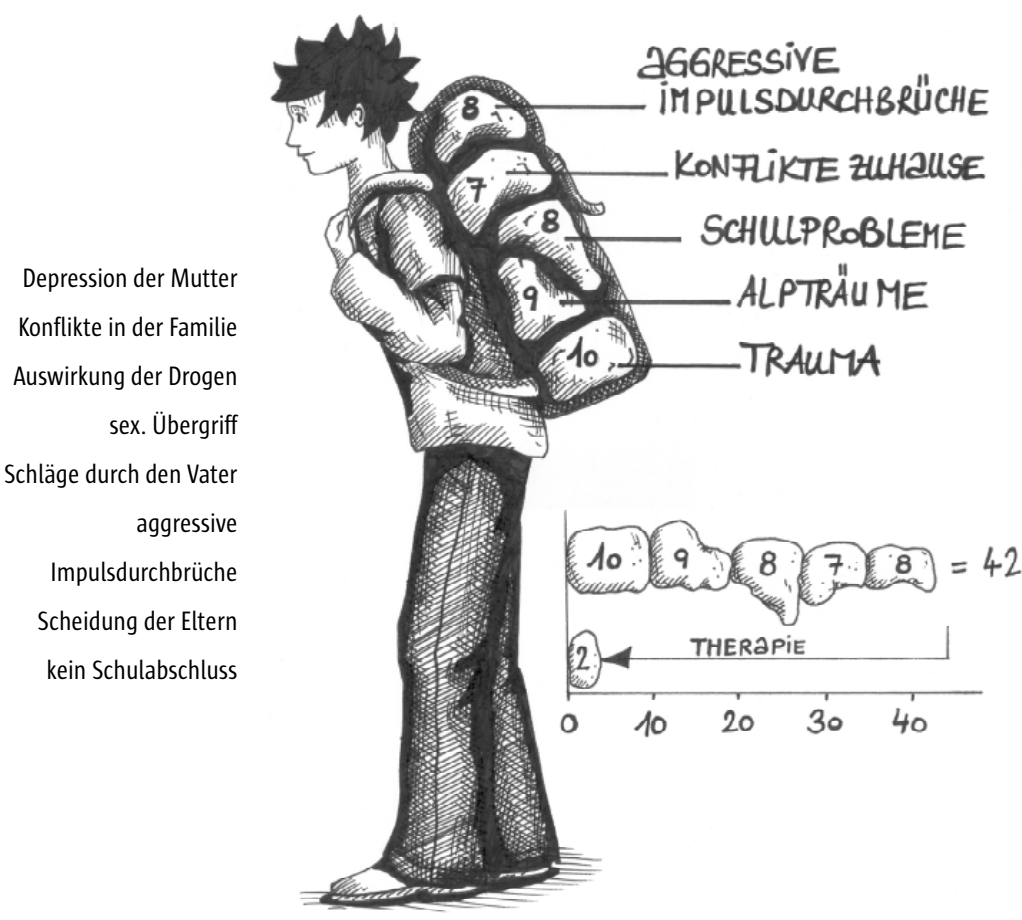

Abb. 5 „Rucksackbild“ mit Belastungsfaktoren und SUD-Skala 


\subsection{Fragen an einen potenziellen Täter}

In der folgenden Liste sind einige Fragen für ein Erstgespräch aufgeführt.

\section{Fragen an einen potenziellen Täter}

- Wir machen uns Gedanken über Dich. Hast Du eine Vorstellung, weshalb wir dieses Gespräch führen?

- Was glaubst Du, warum wir uns Gedanken über Dich machen?

- Wir haben gehört, dass Du eine schwere Zeit durchlebst?

- Wie geht es Dir und wie siehst Du Dich selbst?

- Wie ist es Dir in der letzten Zeit in der Schule ergangen?

- Fühlst Du Dich als Opfer und bist Du verzweifelt?

- Fühlst Du Dich von Deinen Mitschülern ausgegrenzt und unerwünscht?

- Hast Du deswegen Rachefantasien entwickelt?

n Welche Pläne hast Du entwickelt?

n Wer wäre am meisten überrascht gewesen, wenn Du Dein Ziel erreicht hättest?

- Was sollte werden, wenn Du Dein Ziel erreicht hättest?

- Was hast Du Deinen Mitschülern gesagt und womit hast Du ihnen vielleicht gedroht?

- Was wolltest Du ihnen eigentlich damit sagen?

- Hattest Du das Gefühl, Deine ausweglose Situation nur mit Waffengewalt beenden zu können?

- Worin siehst Du den Unterschied darin, Dich allein zu töten oder noch andere Menschen mit in den Tod zu nehmen?

- Was glaubst Du, was Deine bedrohten Mitschüler über Dich denken?

- Was magst Du nun tun, nachdem Du den Mitschüler bedroht hast?

- Welche Hilfen könntest Du akzeptieren, um von Deinem Vorhaben abzusehen?

- Was sind Deine Stärken und Fähigkeiten?

- Würdest Du Dich auf die Beantwortung eines Fragebogens zu Deinen Gewaltfantasien einlassen?

- Gibt es Personen, die wir in unser Hilfsangebot einbeziehen sollten?

- Was würde es für Dich bedeuten, ein Medienstar zu werden?

- Wie groß ist Dein Wunsch nach Unsterblichkeit?

Die vielleicht interessantesten Hinweise darauf, was in den Köpfen von jugendlichen Amokläufern und School Shootern vorgeht, liefert jedoch eine genaue Analyse ihrer Gewaltfantasien. Nach Lempp (2006) können sich solche Fantasien festsetzen und zu einer Überidentifikation führen, die einen vorübergehenden Realitätsverlust zur Folge haben.

W. Huck (2009) entwickelte einen ausführlichen Fragebogen zu Gewalterfahrungen und Gewaltfantasien - insbesondere für strafrechtliche Begutachtungen. Er ermöglicht es, einen Zugang zu den destruktiven Gewaltvorstellungen 
der Jugendlichen zu bekommen. Der Fragebogen wurde unter anderem auch bei jugendlichen Intensivtätern der JVA Iserlohn mit einem hohen Gewaltpotenzial erprobt. Eine Validierung liegt noch nicht vor.

Abbildung 6 illustriert, unter welchem Druck potenzielle Amoktäter stehen.
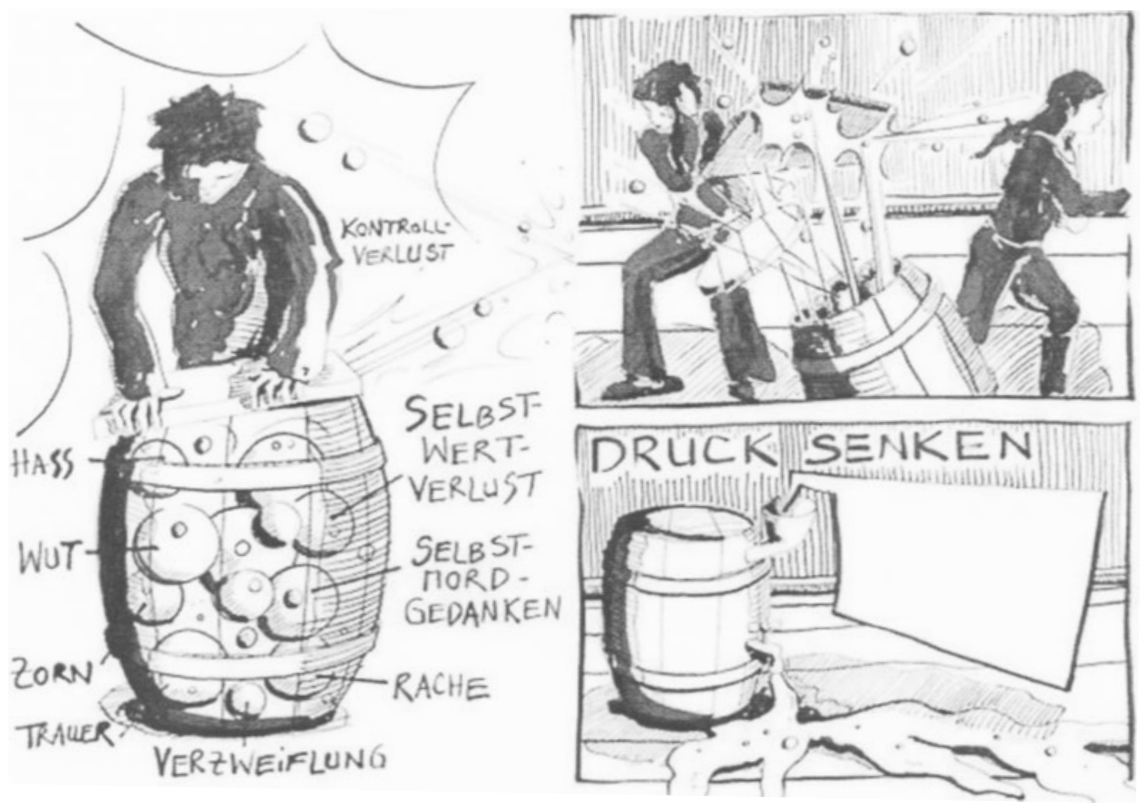

Abb. 6 „Druckkessel“ voller Aggressionen

\subsection{Auszüge aus dem Fragebogen „Gewalterfahrungen und Gewaltfantasien“}

\section{Satzergänzungstest}

- Wenn ich an alle meine bisherigen Demütigungen denke, ...

- Als ich mich gemobbt oder ausgeschlossen fühlte, ...

- Wenn ich Wut in mir spüre und sich mein Denken nur noch auf diese Wut konzentriert, ...

- Wenn ich mich in meinem Selbstwertgefühl verletzt fühle, ...

- Wenn ich den Eindruck habe, dass ich meine Ziele nicht verwirklichen kann, ...

- Bevor ich vernichtet und ausgelöscht werde, ...

- Wenn ich den Wunsch in mir verspüre, nicht einer unerträglichen Situation fliehen zu können, ...

- Als ich schon mal aggressiv werden wollte, rettete mich vor der Ausführung, ... 
- Wenn ich meinem Leben durch Selbstmord einen Sinn geben könnte, ...

- Wenn ich mir vorstelle, einen Blutrausch zu erleben, ...

- Wenn ich Lust verspüre, „Ballerspiele“ in die Tat umzusetzen, ...

- Wenn ich mir ausmale, ein „Held“ werden zu können, ...

- Wenn ich Interesse für Tarnkleidung und Waffen spüre, ...

- Wenn ich alle Macht der Welt haben würde, ...

\section{Weitere Fragen}

- Hast schon einmal mit anderen über Deine Gewaltfantasien gesprochen?

- Gibst Du manchmal absichtlich Hinweise über Deine aggressiven Pläne, um die Reaktion der anderen zu testen?

- Suchst Du Kontakt zu Gleichaltrigen im Internet, die ihre Gewaltfantasien ausleben wollen?

- Möchtest Du Deine Fantasien allein oder mit anderen ausleben?

- Wer sind Deine stärksten Feindbilder?

- Was waren die größten Belastungen, die Du bisher erlebt hast?

- Würdest Du über Deine geplanten Gewalttaten mit jemand reden, damit er Dir hilft, von Deinem Vorhaben Abstand zu nehmen?

- Bekommen Deine Eltern, Geschwister und Freunde von Deinen Gewaltfantasien etwas mit?

- Hast Du schon Waffen oder andere gefährliche Gegenstände gesammelt?

- Stellst Du Dir vor, in der Zeitung, im Fernsehen, Radio oder Internet durch eine Gewaltaktion bekannt zu werden?

- Hast Du Listen von Menschen angelegt, die Du am liebsten ausschalten und vernichten möchtest?

- Gibt es bestimmte Vorbilder von Gewalttätern, die Deine Gewaltfantasien beeinflussen?

- Fällt es Dir schwer, Dich in andere einzufühlen?

- Hast Du häufig Rachegedanken?

- Hast Du das Gefühl, dass Du niemals die nötige Anerkennung bekommst?

- Welchen Personen könntest Du Dich anvertrauen und über Deine Schwierigkeiten sprechen?

- Ist Selbstmord in Deiner Situation ein Ausweg?

- Welche Hilferufe hast Du ausgesandt, die aber nicht beachtet wurden?

In dieser Eingangsphase und „Auftragsklärung“ geht es um eine umfassende Diagnostik, um den Einbezug von Bezugspersonen aus der Familie und aus der Schule, des schulpsychologischen Dienstes, bisher konsultierter Therapeuten und Ärzte, des Jugendamts, des Schulamts, der Polizei und der Staatsanwaltschaft für den Fall einer stationären gutachterlichen Untersuchung nach $\mathbb{1 2 6}$ StGB. 
Nicht nur eine individuelle Psychotherapie ist für potenzielle Täter angezeigt, sondern auch eine Beteiligung von Familie und Schule, um möglichst viele der ihn beeinträchtigenden Faktoren zu beseitigen (s. Box).

\section{Konkrete Unterstützung und Hilfsangebote}

- „Auszeit“ und „Ortswechsel“ zur Entlastung und Entstressung der potenziellen Täter und der potenziellen Opfer sowie zum Schutz der Öffentlichkeit, aber letztlich auch zum Schutz des Täters vor "Medienhatz"

- Erarbeitung eines Verständnisses über Gründe der geplanten Tat und der damit verbundenen destruktiven „Dynamik“, seines Kontrollverlusts und der Folgen für inn selbst und seiner Mitwelt

- Darstellung des Tatplans mit dem Erkennen des „Point of no Return“ und dem damit verbundenen Verlust der Selbstkontrolle

- Analyse der negativen und destruktiven Überzeugungen

- Fragen nach bisher erlebten positiven Ausnahmen, Fähigkeiten und Stärken mit Erstellung eines „Ressourcenrucksacks“ im Vergleich zum „Belastungsrucksack"

- Erarbeitung neuer, selbstentwickelter Perspektiven

- Hilfen bei der Entscheidungsfindung für eine Therapie

- Klärung des therapeutischen „Setting“ und des zukünftigen Entwicklungsrahmens

- Einbezug der Bezugspersonen aus Familie, Freundschaften, Schule, insbesondere bei der Klärung eines Schulwechsels oder einer möglichen Rückkehr in die frühere Klassengemeinschaft, Entwicklung eines besseren Klassenklimas

- Aufbau einer alternativen Freizeitgestaltung und eines neuen sozialen Netzwerkes

In den Gesprächen sollte man sich nach den Vorstellungen und Plänen des Jugendlichen erkundigen oder sie gemeinsam erarbeiten. Entscheidend ist dabei, dass er „sein Gesicht wahren“ kann und man ein echtes Interesse an seinem Schicksal zeigt. Auch sollte Kontakt zu ihm und seiner Familie aufrechterhalten werden. Den Eltern sollte der Ernst der Lage angemessen dargestellt und das Interesse an einer gemeinsamen Lösung der Krise vermittelt werden.

Nach Meinung von Swenson und Henggeler (2004) sind pädagogische Interventionen, die auf das soziale Umfeld von Jugendlichen mit Störungen des Sozialverhaltens und dissozialen Entwicklungen einwirken, erfolgreicher als allein individuelle Interventionen. In verschiedenen Wirksamkeitsstudien konnte im Vergleich zu Kontrollgruppen eine Abnahme der Rückfallhäufigkeit bis zu 70\% dargestellt werden. Damit war auch eine höhere Wahrscheinlichkeit für eine Wiederaufnahme des Schulunterrichts, für verbesserte familiäre Beziehungen und Reduzierung des Drogenmissbrauchs verbunden. 
Falls vorgesehen ist, ein Kind oder Jugendlichen wieder in den alten Klassenverband zu integrieren, sollten Klassenlehrer, schulpsychologischer Dienst und Familie mit dem behandelnden Therapeuten vorbereitende Gespräche führen, um die Integration zu fördern. Danach ist zu entscheiden, ob dem potenziellen Täter konkrete Warnungen ausgesprochen und entsprechende Konsequenzen angekündigt werden. Entscheidend ist dabei auch, welche „Coping-Strategien“ sich der Jugendliche im Umgang mit seinen aggressiven Fantasien und Impulsen erarbeitet hat.

\subsection{Behandlung und therapeutische Möglichkeiten}

Konkrete Ergebnisse von Behandlungen von School Shootern sind bisher bis auf einen früheren Beitrag von W. Huck (2009) nicht publiziert worden.

Die Täter in Littleton und Winnenden wurden medikamentös behandelt. In den von Hoffmann et al. (2009) erfassten Fällen waren zwei von sieben Täter in Behandlung. Auch der Täter Tim K. aus Winnenden wurde in einer Kinderund Jugendpsychiatrischen Klinik ambulant behandelt. Die Therapie potenzieller Täter dient gleichzeitig der Prävention weiterer Taten. Sie sollte auf die individuelle Entwicklungsgeschichte des Betreffenden abgestimmt sein (Strüber et al. 2006, 2008). Im Sinne von lösungs- und ressourcen-orientierten Ansätzen gilt es, den potenziellen Täter darin zu begleiten, selbst gesetzte Ziele zu entwickeln, für die es sich lohnt, sich anzustrengen, um seinen Selbstwert zu stärken und in seiner Persönlichkeit zu reifen.

Lösungsorientierte Fragen zu möglichen Zielen:

- Angenommen, Deine hohen Belastungswerte würden im Verlauf einer Therapie auf einen SUD-Wert von 2 abfallen, wie würdest Du Dich dann fühlen?

- Wer würde in Deinem Umfeld als erste Person diese Veränderung bemerken?

- Woran würde die Person Deine Veränderungen erkennen?

- Wenn Du diese selbstbestimmten Ziele erreicht hättest, wärst Du dann stolz auf Dich?

- Welche Person wäre außerdem noch stolz auf Dich?

- Wie lange benötigst Du für eine Entscheidung, Dich auf eine Therapie einzulassen?

Die Entwicklung einer lebensbejahenden Einstellung und freundschaftlichen sowie vertrauensvollen Beziehungen sind zwei der wichtigsten Schutzfaktoren, die eine Umsetzung gewalttätiger Fantasien verhindern. Der potenzielle Täter muss Empathiefähigkeit entwickeln und lernen, seine Frustrationen angemessen zu bewältigen und gewaltfreie Handlungsoptionen in Konfliktsituationen zu entdecken und zu erproben. 
Das schwierigste in der ersten Phase der Motivation der potenziellen Täter und seiner Bezugspersonen ist die Herstellung eines tragfähigen Arbeitsbündnisses und die Erarbeitung eines Behandlungsauftrags. Außerdem müssen verschiedene „Rahmenbedingungen und Eckpunkte“ mit dem Jugendlichen ausgehandelt werden (s. Abb. 7). Damit wird für alle Beteiligten eine „Transparenz“ des Behandlungsprozesses ermöglicht. Gleichzeitig sind dies auch „vertrauensbildende Maßnahmen“, durch die der Jugendliche auch eine neue Erfahrung von Verlässlichkeit machen kann.

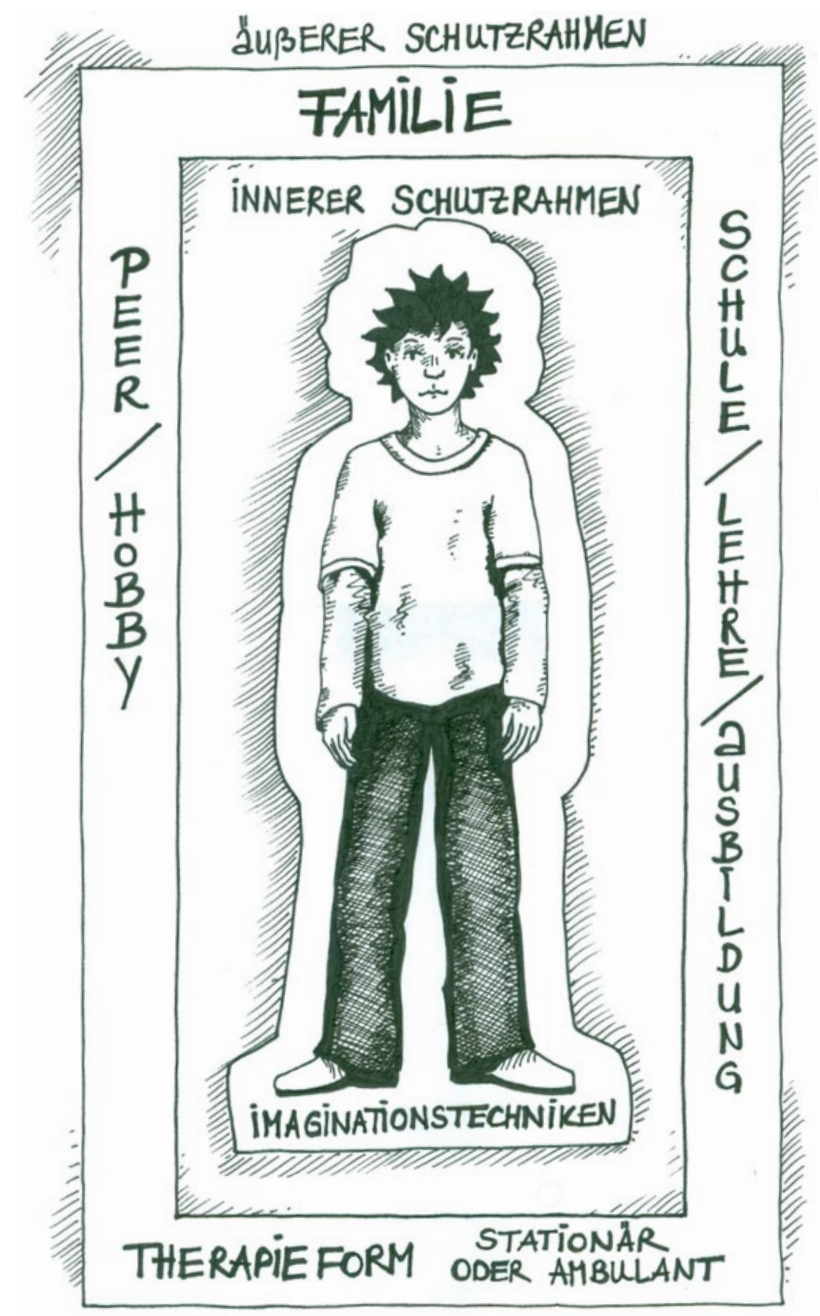

Abb. 7 „Rahmen“ mit den relevanten Bezugspersonen 


\section{Die Jugendlichen brauchen bei der Behandlung zunächst „externe“ Kontrollinstanzen, um eine „interne“ Kontrollinstanz und eine Gewis- senstruktur aufzubauen.}

\subsection{Wer bin ich eigentlich? Die Frage nach der Identität}

Für E. Erikson (1964) besteht das Kernproblem der Identitätsbildung in der Fähigkeit des Ichs, angesichts des wechselnden Schicksals „Gleichheit und Kontinuität“ aufrechtzuerhalten. Nach Keupp et al. (2008) wird Identität „unter den Bedingungen der Postmoderne“ nicht mehr als Entstehung eines „inneren Kerns“, sondern als „Prozessgeschehen beständiger alltäglicher Identitätsarbeit“ und als permanente „Passungsarbeit“ zwischen äußerer und innerer Welt thematisiert. Jeder Jugendliche wird zu seinem eigenen „Baumeister“ seines eigenen „Beziehungsnetzwerkes“. Ansonsten besteht für ihn die Gefahr, die „Lebensfeindlichkeit sozialer Wüsten“ und das Gefühl der Ausgeschlossenheit zu erleben.

Nach Luhmann (zit. nach Reemtsma, 2002 und 2009b) ist Identitätsbildung nicht einfach der „Vollzug eines biologischen Programms“. Er interpretiert das Gewissen als eine „Funktion im Dienst der Identitätsbildung“. Es handelt sich dabei um einen Entwicklungsprozess, der eng damit zusammenhängt, wie in einer bestimmten, gesellschaftlichen Epoche die personale Entwicklung definiert wird (Keupp et al. 2008). Es ist ein subjektiver Konstruktionsprozess, „in dem Individuen eine Passung von innerer und äußerer Welt" versuchen. In diesem Prozess müssen sie sich mit folgenden Fragen auseinandersetzen.

\section{Fragen zum Identitätsfindungsprozess}

- Wie kann ich in einer sich ständig wandelnden und widersprüchlichen Welt eine „stimmige Passung“ herstellen, die mir hilft, mich in meiner Umwelt zurechtzufinden und handlungsfähig zu sein?

- Gelingt es mir, eigene Vorstellungen und Handlungskonzepte zu entwerfen?

- Was ist dabei für meine eigene psychische Gesundheit von zentraler Bedeutung?

- Flößen mir die wahrgenommenen Widersprüche in der Gesellschaft und als fremd Empfundenes Angst ein, die Kontrolle zu verlieren?

Auf der „Suche nach Verortung und Beheimatung“ gilt es, im therapeutischen Prozess mit den potenziellen Tätern „regressive Tendenzen“ aufzuspüren, ihnen zu helfen, vertrauensvolle Kontakte zu knüpfen, ohne Angst haben zu müssen, erneut entwertet zu werden. Deshalb ist das therapeutische Gespräch so zu führen, dass die Jugendlichen nicht mit einer „niederschmetternden“ Kritik rechnen müssen, sondern die Hinweise der Therapeuten ein für sie nützliches Feedback erleben können. 


\subsubsection{Resilienz und Schutzfaktoren}

$\mathrm{Zu}$ aggressiven Regungen der Kinder und Jugendlichen kommt es insbesondere dann, wenn ihre Grundbedürfnisse physiologischer Art, nach Sicherheit, nach Zuwendung und nach Beachtung ihrer individuellen Entwicklung vernachlässigt werden.

Calkins et al. (2007) ermittelten in ihrer Verlaufsuntersuchung an 441 Kindern im Vorschulalter Resilienzfaktoren, welche Vorhersagen über den Grad an externalisierendem Verhalten zulassen. Kinder, die über diese Fähigkeit verfügen, ecken im gesellschaftlichen Alltag weniger an und haben mehr schulischen und beruflichen Erfolg. Vorhandene Resilienz und Schutzfaktoren sowie Coping-Strategien werden im Verlauf der Behandlung für eine positive Entwicklung genutzt. Resiliente Heranwachsende bewältigen Entwicklungsrisiken ohne kognitive, emotionale und soziale Beeinträchtigungen und zeigen sich selbstbewusst und ausgeglichen, wenn sie ein positives Selbstkonzept entwickelt haben. Dieses weist eine angemessene Selbsteinschätzung und eine eigene Kompetenz zum Erleben von Selbstwirksamkeit auf (Lösel 1999; Rentschler 2010).

Im Sinne der Salutogenese geht es im therapeutischen Prozess um die Beachtung der bestehenden Schutzfaktoren (s. Box), Ressourcen und intakter Persönlichkeitsanteile.

\section{Schutzfaktoren}

- Besteht noch eine gute Beziehung zu einer primären Bezugsperson?

- Gibt es noch im erweiterten Familienumfeld tragfähige und kompensatorische Beziehungen?

- Zeigt zumindest ein Elternteil einen konsequenten Erziehungsstil?

- Nehmen Eltern am Leben des Jugendlichen teil?

- Besitzt der Jugendliche ein gutes und ausgeglichenes Intelligenzprofil?

- Hatte der Jugendliche vor seiner Krise ein robustes, aktives und kontaktfreudiges Temperament?

- Gibt es für die Zeit vor der Krise Hinweise auf ein sicheres Bindungsverhalten?

- Gibt es Hinweise auf Phasen mit einem positiven Selbstwertgefühl?

- Wie ausgeprägt sind seine externalen und internalen Kontrollüberzeugungen?

- Bestand vor der Krise ein wenig konflikthaltiges, offenes und auf Selbstständigkeit orientiertes Erziehungsklima?

- War der Jugendliche früher gelegentlich in Jugendgruppen integriert?

- Hat er bisher ausreichende Entwicklungsstimuli außerhalb der Familie erhalten?

- Wie ausgeprägt waren vor der Krise seine subjektiven wie objektiven schulischen Chancen und Perspektiven? 
Nach Keupp et al. (2008) ist ein angemessenes Kohärenzgefühl die wesentliche Grundlage für eine erfolgreiche Bewältigung von Stresssituationen. Es stellt dabei keinen spezifischen Bewältigungsstil dar, entfaltet aber seine Wirkung durch Mobilisierung von Widerstandsfähigkeiten und durch flexible Auswahl von Strategien, die für die Bewältigung am erfolgreichsten erscheinen. Die subjektive Zuweisung von „Sinn“ ist nach Keupp et al. (2008) eine Hauptfunktion bei der Mobilisierung von Ressourcen und der flexiblen Wahl von Bewältigungsstrategien. In diesen Prozessen tauchen viele Schuldgefühle und Fragen nach der eigenen Verantwortung auf. Personen mit einem schwachen Kohärenzgefühl geben die Schuld eher anderen, worin sich oft „ineffektive Abwehrmechanismen“ ausdrücken. Jugendliche mit einem hohen Kohärenzgefühl unterscheiden sich von anderen, wie sie Stressoren bewältigen, und auch darin, wie es ihnen gelingt, eigene „Erfahrungsräume“ herzustellen, die für sie gesundheitsfördernd sind.

\subsubsection{Die narrative Konstruktion von Kohärenz}

Keupp et al. (2008) formulierten die These, dass Kohärenz für die alltägliche Identitätsarbeit von Menschen eine zentrale Bedeutung besitzt, deren Fehlen zu schwerwiegenden gesundheitlichen Konsequenzen führt. Kohärenz wird über individuelle Geschichten, „Narrationen“, erzeugt. Wenn durch eine krisenhafte Zuspitzung die ,innere Kohärenz“ verlorengegangen ist, muss man dem Jugendlichen helfen, diese durch eigene Narrationen erneut zu finden, aufzubauen und zu stabilisieren.

Meuter (1995) mit seiner philosophischen Dissertation und Boothe (2011) mit ihrem Lehrbuch über Narrationen rückten das Konzept der „narrativen Identität“ ins Zentrum der wissenschaftlichen Auseinandersetzung. Erzählungen und Geschichten waren und bleiben die einzigartige menschliche Form, das eigene Erleben zu ordnen, zu bearbeiten und zu begreifen. Erst in einer Geschichte, in einer geordneten Sequenz von Ereignissen und deren Interpretation gewinnt das Chaos von Eindrücken und Erfahrungen, dem jeder Mensch täglich unterworfen ist, eine gewisse Struktur, vielleicht sogar einen Sinn (Ernst 1996).

Nach Keupp et al. (2008) ist Identitätsarbeit ein Prozess, bei dem vergangene, gegenwärtige und zukunftsbezogene Selbsterfahrungen unter verschiedenen Identitätsperspektiven reflektiert und zu „Teilidentitäten“ zusammengefasst werden. Der Identitätsprozess ist voller Ambivalenzen, Spannungen und Widersprüche. Identitätsbildung als „Passungsprozess“ meint nicht, diese Differenzen zu harmonisieren, sondern sie in ein für das Subjekt „lebbares Beziehungsverhältnis“ zu bringen. Erzählend organisiert der Jugendliche die Vielgestaltigkeit seines Erlebens in einen Verweisungszusammenhang. Die „narrativen Strukturen“ sind keine ausschließliche Eigenschöpfung des Individuums, sondern im sozialen Kontext verankert und von ihm beeinflusst, sodass ihre Ge- 
nese und ihre Veränderung in einem komplexen Prozess der Konstruktion sozialer Wirklichkeit stattfinden. „Selbsterzählungen“ machen eine Person verstehbar für andere. Was jemandem widerfährt, wie er die Welt sieht und wie er Erlebtes bewertet, das alles gibt ihm für ihn selbst und für andere eine Gestalt. Die „Konstruktionsarbeit“ an diesen Selbstgeschichten ist ein ständiger Prozess (s. Box).

\footnotetext{
„Konstruktionsarbeit“ an den eigenen Geschichten nach Keupp et al. (2008)

- Wie können Jugendliche eine eigene Handlungsfähigkeit entwickeln?

- Welche Bedeutung kommt der Erfahrung sozialer Anerkennung und Zugehörigkeit für die Identitätsbildung zu?

- Wie hängt das Ergebnis der Identitätsarbeit von den psychischen, sozialen, kulturellen und materiellen Ressourcen einer Person ab?

- Wie stellen sich Jugendliche mit ihren Identitätsvorstellungen auf gesellschaftliche Rahmenbedingungen ein?

- Wie kann der Jugendliche Anerkennung von anderen erreichen?
}

Der Erfolg in einem Prozess der Identitätsbildung hängt wesentlich von Anerkennung und angemessenem Wahrgenommenwerden und positiver Bewertung durch andere ab. Außerdem spielt Selbstanerkennung und positive Selbstbewertung eine entscheidende Rolle. Erst wenn alle Elemente erfüllt sind, kann eine Selbstfindung ihre Wirkung entfalten. Ansonsten bleibt die Identitätsbildung unvollständig und führt zu ständigem Selbstzweifel. Mit Erleben von Kohärenz, Anerkennung und Authentizität beschreiben Keupp et al. (2008) „drei Modi alltäglicher Identitätsarbeit“, die als Indizien für eine „gelungene Identität“ bezeichnet werden können.

In einem Identitätsprozess kommt es auch zu einer Auseinandersetzung mit bis nicht ausgelebten Sehnsüchten. So verbirgt sich hinter dem „Traum vom Berühmtsein" potenzieller Schul-Amoktäter und School Shooter häufig die Sehnsucht nach Anerkennung, Beachtung, Zugang zu einem anerkannten sozialen Sein und nach Zugehörigkeit. Sie möchten nicht übersehen, verlacht oder ausgegrenzt werden (Baumann 2009; Bourdieu 2001).

Nach Baumann (2009) ist ein wichtiges Kennzeichen der heutigen Konsumgesellschaft - so sorgfältig verborgen und verheimlicht es auch ist - die „Verwandlung von Konsumenten in Waren“. Ausschließlich als Objekt betrachtet, fühlen sich die Jugendlichen missachtet und verkannt (Lévinas 1991).

Buber (1986) entwickelte die fundamentale These: „Am Anfang war Beziehung“. Wenn sich jedoch durch Ausbildung von Parallelwelten „Ver-gegnung“einstellt, hat dies oft verheerende Wirkung. Der Dialog zwischen Eltern und den Jugendlichen, zwischen Lehrern und Schülern beinhaltet, in ihren Antworten „Ver-antwort-lichkeit“, indem sie für einander Verantwortung übernehmen. Der Status des „zwischen“ ist nach Buber (1989) ein „Seins-Modus“ der Mit-Anwesen- 
heit und des Mit-Seins. Die Begegnung findet „zwischen“ Personen statt. Der Dialog funktioniert nicht als „Synthese“ der Beziehung, sondern als deren eigentliche Entwicklung. Ein solches soziales Zusammenleben kann nur auf gegenseitigem Vertrauen basieren, sonst müssten Therapeuten wie Patienten „dauernd aufder Hut“ sein. Ihr Kontakt würde auf wenige Handlungsoptionen beschränkt bleiben. In familientherapeutisch orientierten Gesprächen wird in der Phase der Adoleszenz immer wieder ein erheblicher „Autonomie-Abhängigkeitskonflikt“ deutlich. Die potenziellen Täter konnten in ihrer Autonomieentwicklung oft keine konstruktiven Abgrenzungs- und Unabhängigkeitsbestrebungen entwickeln.

Potenzielle Täter sind oft in hochambivalenter Weise an Bezugspersonen gebunden, was zu erheblichen Loyalitätskonflikten führt. Dadurch kommt es oftmals zum Scheitern pädagogischer und therapeutischer Bemühungen. Folgende Behandlungsziele sollten im Mittelpunkt der Behandlung potenzieller Amoktäter stehen.

\section{Wichtige Behandlungsziele von Amokläufern und School Shootern}

- Entwicklung empathischer Fähigkeiten

- Entwicklung von Strategien für angemessenen Konfliktlösungen und zur Reduktion von Stress

- Stärkung des Selbstwertgefühls

- Erfahrung von eigener Wirksamkeit

- Aufarbeitung der erlittenen Gewalterfahrung

- Entwicklung von Selbstkontrolle

- Aushandeln und Akzeptieren von Regeln

- Aufbau sozialer Kompetenzen

- Entwicklung eines Notfallplans gegen Wiederbelebung von Gewaltfantasien

- Identifikation wichtiger Konfliktfelder

- Entwicklung von Ausdauer und Verzicht auf sofortige Bedürfnisbefriedigung

- Erlernen von Rücksichtsnahme, Achtsamkeit gegenüber anderen

- Lernen aus Fehlern und Aufbau einer neuen "Fehlerkultur“

- Aufbau eines positiven Verhältnisses zur Schule, zu Mitschülern und Lehrern

Fthenakis (2004) zog aus seiner Analyse der bisherigen Amoktaten die Schlussfolgerung, dass zur Prävention solcher Ereignisse „einfühlsame, wertschätzende und unterstützende Beziehungen zwischen Lehrkräften und Schülern" notwendig sind. In Deutschland gibt es seiner Meinung nach diesbezüglich noch einen enormen Nachholbedarf. So ergab das „Kinderbarometer der LBS-Initiative“ von 2007, dass 20\% der befragten Schüler zwischen 9 und 15 Jahren darunter leiden, dass ihre Lehrer sie bloßstellen, anstatt sie ernst zu nehmen und ihr Selbstwert- 
gefühl stärken. Der wichtigste Faktor für eine gesunde psychische Entwicklung ist nach wie vor die Familie mit Erfahrungen einer sicheren Bindung zu den Eltern, deren Anerkennung und Wertschätzung. Nur mit einem positiven Selbstwertgefühl können Kinder und Jugendliche Verantwortung übernehmen und Konflikte adäquat bewältigen.

\subsubsection{Fortbildung für Lehrer}

Die für Schulen zuständigen Behörden haben in der Zwischenzeit empfohlen, Schulteams für Gewaltprävention und Krisenintervention zu bilden, um sich im Ernstfall angemessen verhalten zu können. Diesen Prozess sieht der Verfasser aufgrund der Erfahrungen, die er im Rahmen seiner aktuellen „Vernetzungsarbeit“ mit verschiedenen schulpsychologischen Diensten sammelte, noch nicht abgeschlossen. Die Bildung der Teams für Beratung, Prävention und Krisenintervention muss weiter verfolgt werden. Krisenteams sollen präventive und Krisen bewältigende Strukturen aufbauen. Inzwischen bieten verschiedene Fortbildungseinrichtungen, die Polizei und örtliche Schulpsychologen Hilfestellungen zur Bildung von Krisenteams an und weisen sie in die Umsetzung von Notfallplänen ein. Lokale Kompetenzteams führen Veranstaltungen zur Gewaltprävention in Schulen durch. Zu ihren Fortbildungsschwerpunkten gehört der Bereich „Entwicklung sozialer Kompetenz“ für alle Schulformen und Schulstufen. Lehrerverbände und Gewerkschaften stellen ebenfalls Fortbildungsmöglichkeiten zur Verfügung.

\subsubsection{Das Achten auf die eigene Psychohygiene}

Nach einem Amoklauf sollte das traumatisierende Ereignis mit allen Beteiligten aufgearbeitet werden. Zunächst geht es um eine sofortige psychologische Betreuung der Betroffenen, um posttraumatische Belastungsstörungen möglich zu verhindern. Später geht es um die Vermittlung Betroffener in geeignete Therapien und um Aufarbeitung des Geschehenen und Herstellung einer erträglichen Erinnerung an den Vorfall und die Opfer.

Die Unfallversicherung mit Sitz in Münster vereinbarte mit dem Landschaftsverband Westfalen-Lippe seit dem 1.10.2009, durch seine psychiatrischen Kliniken professionelle Nachsorge im Großschadensfall zur Verfügung zu stellen. Der „Expertenkreis in Baden-Württemberg“ zeigt in seinem Bericht nach dem Amoklauf in Winnenden weitere Aspekte auf. Dabei geht es um den weiteren Ausbau psychosozialer Beratung, um exaktere Information für Ärzte, Psychiater und Psychotherapeuten, um eine angemessene Opferhilfe und um weiterbildendes Katastrophentraining bei Polizei und Feuerwehr. 